\title{
Adsorption and Unfolding of Lysozyme at a Polarized \\ Aqueous-Organic Liquid Interface
}

\section{Supporting Information}

Mahreen Arooj, ${ }^{1}$ Neha S. Gandhi, ${ }^{1}$ Cara A. Kreck, ${ }^{1}$ Damien W.M. Arrigan ${ }^{2}$ and Ricardo L. Mancera $^{1 *}$

${ }^{1}$ School of Biomedical Sciences, CHIRI Biosciences and Curtin Institute for Computation, and

${ }^{2}$ Department of Chemistry and Nanochemistry Research Institute, Curtin University, GPO Box U1987, Perth WA 6845, Australia.

* Author for correspondence: R.Mancera@curtin.edu.au

Tel. +61892661017

Fax. +6189266 2342 




Figure S1: Orientation of HEWL at the start of the simulation and after adsorption at the water/DCE interface. 




(A)

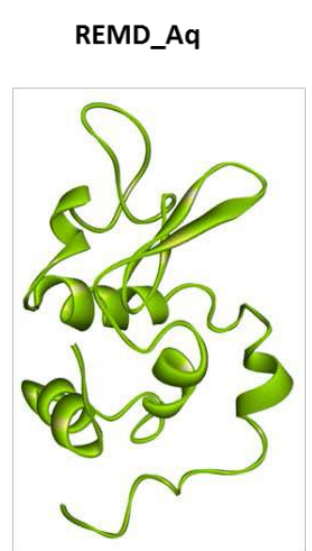

(B)

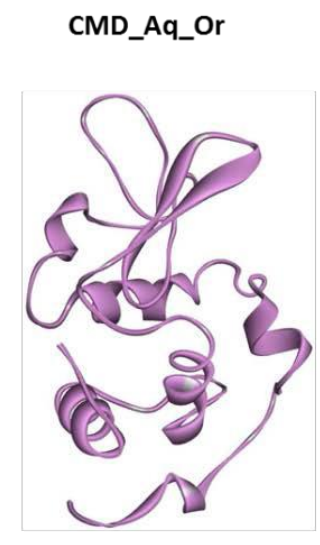

(C)
Superimposed on Crystal Structure

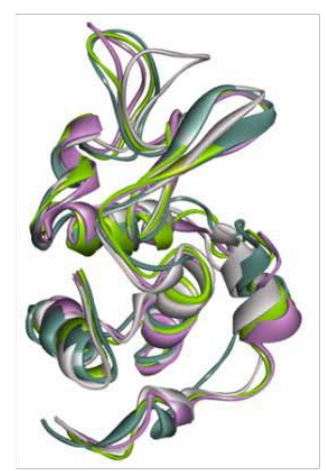

(D)

Figure S2: Representative structures of HEWL from cluster analysis of (A) CMD_Aq, (B) REMD_Aq, and (C) CMD_Aq_Org simulations. Superimposition of these representative structures onto the crystal structure of HEWL is shown in D. 


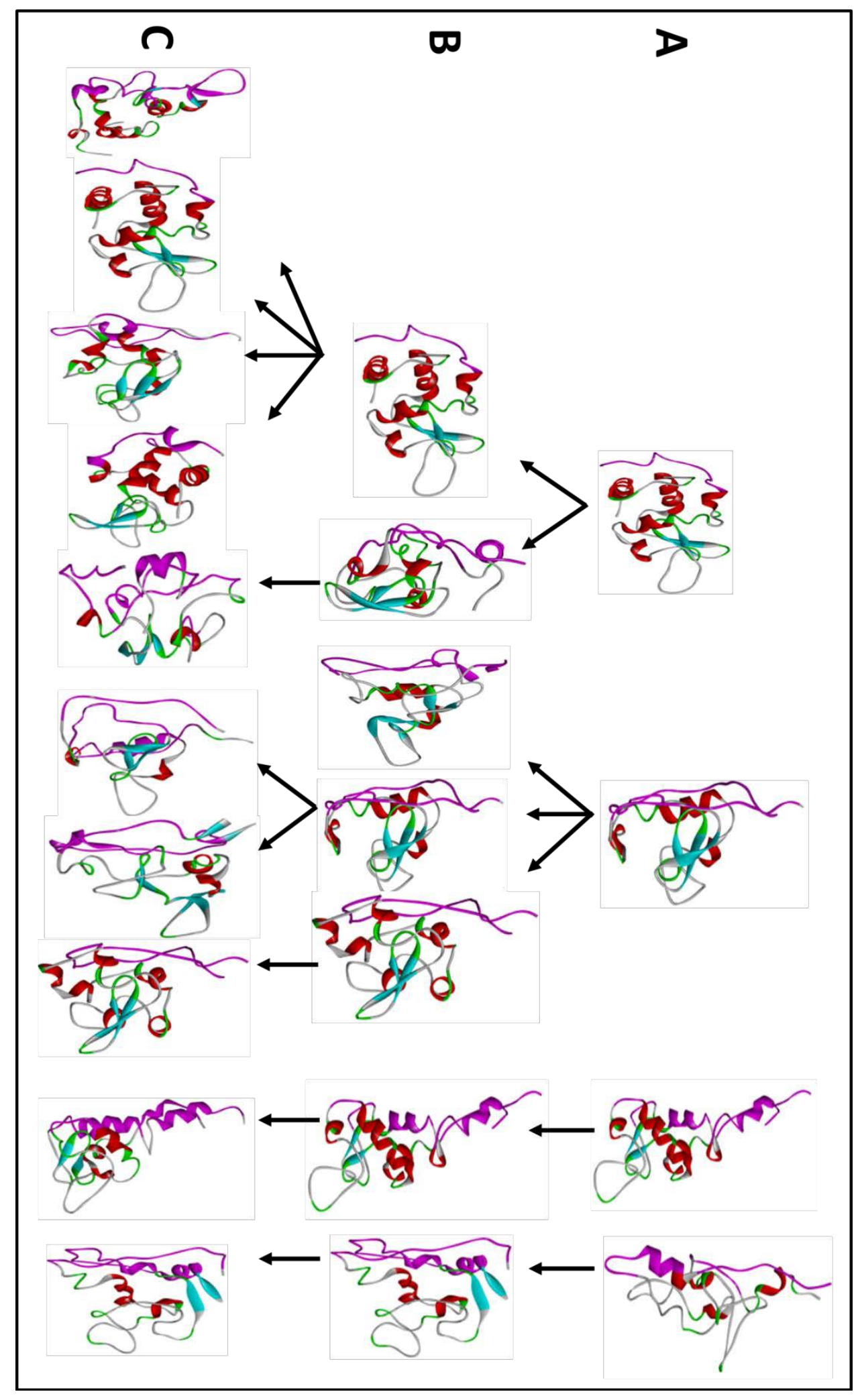

Figure S3: Representative structures of top clusters of HEWL adsorbed at the water/DCE interface (REMD_Aq_Org simulation) obtained with clustering cut-off values of $0.8 \mathrm{~nm}(\mathbf{A})$, $0.7 \mathrm{~nm}(\mathbf{B})$, and $0.6 \mathrm{~nm}(\mathbf{C})$. 


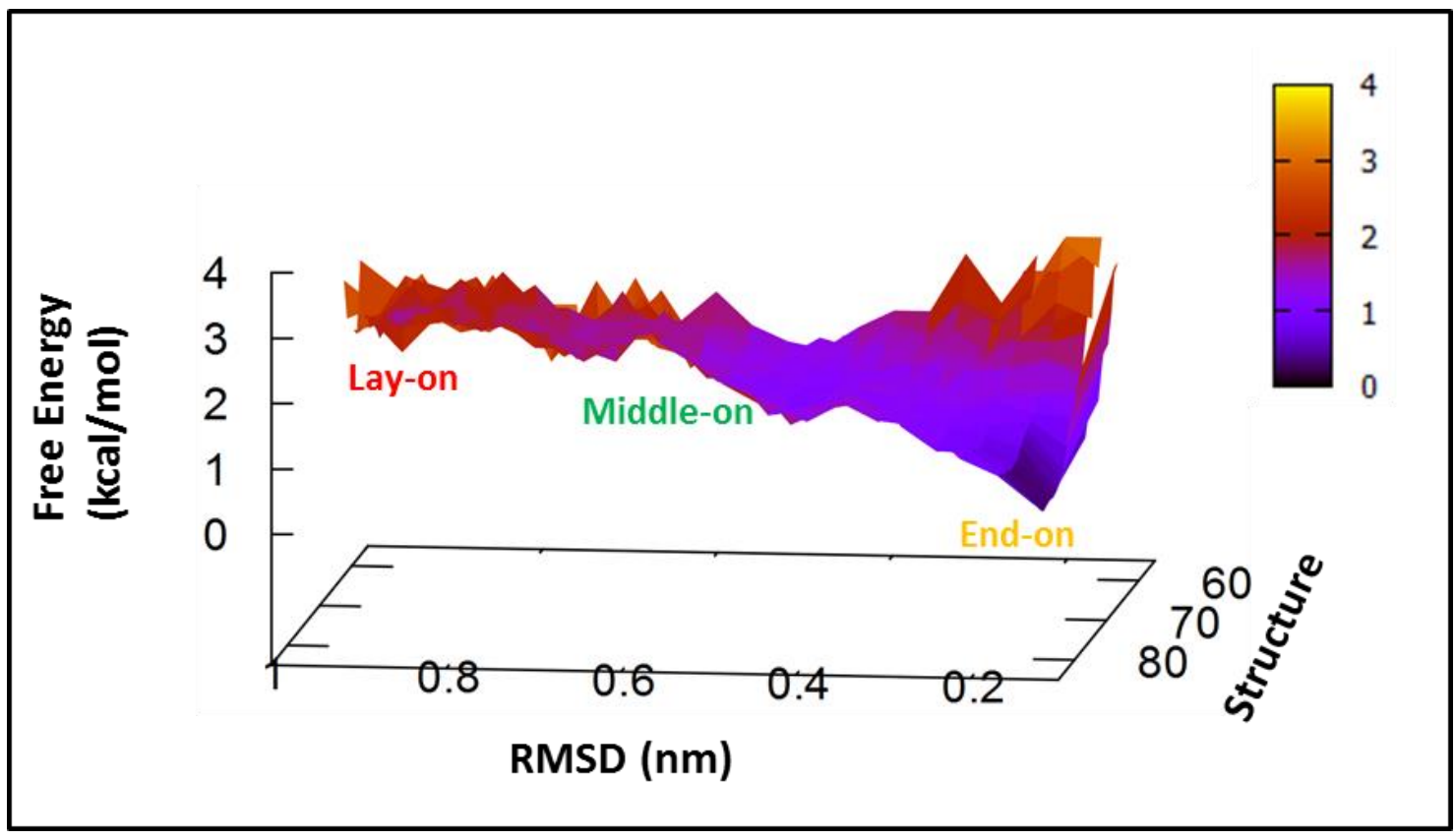

Figure S4: Free energy landscape for the unfolding of HEWL as a function of RMSD and secondary structure content for conformations belonging to the dominant three orientations of the protein at the water/DCE interface. 